\title{
Epacl inhibits PKR to reduce NLRP3 inflammasome proteins in retinal endothelial cells
}

This article was published in the following Dove Press journal:

Journal of Inflammation Research

\section{Youde Jiang \\ Jena J Steinle}

Department of Ophthalmology, Visual and Anatomical Sciences, Wayne State University School of Medicine, Detroit, MI, USA
Correspondence: Jena J Steinle Department of Ophthalmology, Visual and Anatomical Sciences, 9314 Scott Hall, Detroit, MI 48202, USA

Tel + I 313577973

Fax +3135773I25

Email jsteinle@med.wayne.edu
Purpose: Inflammation has been strongly associated with retinal damage in diseases such as diabetic retinopathy. Several studies have reported that high glucose exposure induces damage to the retinal vasculature. We and others have shown that high glucose can activate the NOD-like receptor family, pyrin domain containing family member 3 (NLRP3) pathway, leading to increased levels of cleaved caspase 1 and IL-1 $\beta$ to activate a number of inflammatory pathways in the retina.

Methods: We used retinal endothelial cells grown in normal $(5 \mathrm{mM})$ or high $(25 \mathrm{mM})$ glucose or retinal lysates from endothelial cell-specific knockout mice for exchange protein activated by cAMP 1 (Epac1). Human recombinant protein kinase R (PKR) or C16, a PKR inhibitor, was used on the cells to dissect PKR and NLRP3 signaling.

Results: Using retinal endothelial cells (REC) in high glucose and whole retinal lysates from endothelial cell-specific knockout of Epac1, we demonstrate that Epac1 regulates PKR phosphorylation. Using an Epac1 agonist or PKR inhibition with C16, we demonstrated that loss of PKR resulted in reduced NLRP3, cleaved caspase 1, and IL-1 $\beta$ levels. Furthermore, despite the addition of recombinant human PKR, Epac1 was still able to significantly reduce NLRP3 signaling.

Conclusion: Overall, these studies demonstrated that PKR regulates the NLRP3 inflammasome in REC, and that Epac1 inhibition of PKR can reduce activation of the NLRP3 inflammasome.

Keywords: inflammasome, NLRP3, PKR, retinal endothelial cells

\section{Introduction}

In the past decades, there has been an increasing acceptance of the role that inflammation plays in the diabetic retina. ${ }^{1-5}$ In addition to the countless others, one potential pathway that may mediate retinal inflammation is the inflammasome. The inflammasome is a multiprotein scaffolding complex that contains a member of the NOD-like receptor family, pyrin domain containing family member (NLRP), procaspase 1, and apoptosis-associated speck-like protein containing a CARD, leading to activation of interleukin-1-beta ${ }^{6,7}$ To date, both NLRP1 and NLRP3 inflammasomes have been associated with diabetic retinopathy; ${ }^{7,8}$ however, most work has focused on the NLRP3 inflammasome. Work in humans with various stages of diabetic showed increased NLRP3 and associated inflammasome proteins in vitreous samples, with the largest responses in patients with proliferative diabetic retinopathy. ${ }^{9}$ We have previously reported that exchange protein for cAMP 1 (Epac1) decreased inflammatory mediators in the retinal vasculature, ${ }^{10}$ as well as inhibited the NLRP3 inflammasome. ${ }^{11}$ Our findings in cells grown in high glucose 
agree with work in retinal pigmented epithelium showing increased NLRP3 and inflammasome proteins, as well as in samples from patients with age-related macular degeneration. ${ }^{12}$ Thus, it appears that the NLRP3 inflammasome may be involved in retinal disease. The remaining key question is upstream regulation of the NLRP3 inflammasome.

Protein kinase $\mathrm{R}$ (PKR) may regulate the NLRP3 inflammasome, as PKR deficiency reduced NLRP3, high mobility group box 1 (HMGB1), and IL-1 $\beta$ levels in macrophages. ${ }^{13}$ PKR is activated by stress signals and upon autophosphorylation, it can lead to NFkB activation and ultimately the inflammasome. ${ }^{14}$ PKR can also be activated by protein activator of the interferon-induced protein kinase (PACT), which is encoded by the PRKRA gene in humans. ${ }^{15}$ In addition to PACT, PKR is phosphorylated by dsRNA during viral infection, and PKR may play a role in metainflammation associated with metabolic syndrome. ${ }^{16}$ In mice and monkeys, studies have shown that tumor necrosis factor alpha (TNF $\alpha$ ) can induce PKR, leading to memory impairment. ${ }^{17}$ Once PKR is phosphorylated, it can activate a number of downstream pathways, leading to inflammatory, apoptotic, or autophagic pathways. ${ }^{18}$ Studies using PKR knockout animals have demonstrated that loss of PKR significantly reduced inflammasome actions and inflammatory mediators. ${ }^{19}$ Taken together, a number of stimuli can activate PKR, leading to downstream inflammatory pathways.

In this study, we wanted to investigate upstream regulation of PKR in the retina of Epac1 conditional knockout mice, as well as in retinal endothelial cells (REC) grown in high glucose. We also tested whether Epac1's inhibition of the NLRP3 inflammasome is mediated through PKR actions.

\section{Methods}

\section{Epacl endothelial cells specific $\mathrm{KO}$ mice}

Animal procedures meet the Association for Research in Vision and Ophthalmology requirements and were approved by the Institutional Animal Care and Use Committee of Wayne State University and conform to NIH guidelines. Epac1 floxed mice (B6;129S2Rapgef3 ${ }^{\text {tm1Geno/J }}$ mice) and B6 FVB-Tg (cdh5-cre)7Mlia/ $\mathrm{J}$ Cre mice were purchased from Jackson Laboratories. The Epac1 floxed mice were bred with the cdh5-Cre mice to generate conditional knockout mice in which Epac1 is eliminated in vascular endothelial cells. At 3 months of age, Epac1 floxed and Epac1 Cre-Lox mice were used for these experiments. ${ }^{20,21}$ Euthanasia was performed with drug overdose followed by cervical dislocation. Whole retinal lysates were collected from the mice.

\section{Retinal endothelial cells}

Primary human REC were purchased from Cell Systems Corporation (CSC, Kirkland, Washington). Cells were grown in Cell Systems medium (Complete Medium Formulated at Normal Blood Glucose Level, $5 \mathrm{mM}$ ) supplemented with microvascular growth factors (MVGS), 10 $\mu \mathrm{g} / \mathrm{mL}$ gentamycin, and $0.25 \mu \mathrm{g} / \mathrm{mL}$ amphotericin B (Invitrogen, Carlsbad, CA). Once cells reached confluence, some dishes were moved to Cell Systems High Glucose Medium (25 mM) for a minimum of 3 days prior to experiments. Only dishes prior to passage 6 were used. Cells were starved by incubating in high or normal glucose medium without MVGS for $12 \mathrm{hrs}$ prior to treatments.

\section{Cell treatments}

Some cells in high glucose were treated with the PKR inhibitor, C16 (Tocris, United Kingdom), in varying doses for $16 \mathrm{hrs}$ to determine the optimal dose for future experiments. Additionally, some cells in high glucose were also treated with recombinant human PKR (rhPKR, Novus Biologicals, Littleton, CO). A time course experiment was completed to determine the optimal timing for activation of PKR. Additional cells received an Epac1 agonist (8-CPT-2'-O-Me-cAMP) for $24 \mathrm{hrs}$ at $10 \mu \mathrm{M} .{ }^{11}$ For cells treated with both rhPKR and Epac1, $\operatorname{rhPKR}(0.24 \mathrm{ng} / \mu \mathrm{L})$ was used for $24 \mathrm{hrs}$ prior to Epacl agonist (24 hrs) application.

\section{Western blotting}

Whole retinal lysates from mice or cell culture lysates were collected into lysis buffer with protease and phosphatase inhibitors. Equal amounts of protein separated onto a precast tris-glycine gel (Invitrogen, Carlsbad, CA) were blotted onto the nitrocellulose membrane. After blocking in TBST (10 mM Tris-HCl buffer, $\mathrm{pH}$ 8.0, $150 \mathrm{mM} \mathrm{NaCl}, 0.1 \%$ Tween $20)$ and $5 \%(\mathrm{w} / \mathrm{v}) \mathrm{BSA}$, membranes are treated with Epac1, phosphorylated PKR, total PKR, NLRP3, cleaved caspase 1, and IL-1 $\beta$ (Abcam, Cambridge, MA) or beta-actin (Santa Cruz Biotechnology, Santa Cruz, CA) primary antibodies followed by incubation with secondary antibodies labeled with horseradish peroxidase. A chemiluminescence reagent kit (Thermo Scientific, Pittsburgh, PA) was used to visualize 
antigen-antibody complexes. Images are acquired on an Azure C500 (Azure Biosystems, Dublin, CA) and optical densities were determined using Image Studio Lite software.

\section{ELISA}

An IL-1 $\beta$ ELISA was performed following the manufacturer's instructions with the exception that $120 \mu \mathrm{g}$ protein was loaded into all wells, and the primary antibody was incubated overnight.

\section{Statistics}

Non-parametric Kruskal-Wallis with Dunn's post-hoc tests were used for the cell culture data. One-way ANOVA with Student Newman Keul's post-hoc test was used for animal work. Data are presented as mean + SEM. $P<0.05$ is taken as statistically significant.

\section{Results}

C16 is a potent inhibitor of PKR phosphorylation in REC grown in high glucose. Before we initiated these studies, we wanted to determine if C16 could effectively inhibit PKR in REC as it had done in other cell types. ${ }^{22}$ Figure 1 shows that $\mathrm{C} 16$ was highly effective in reducing PKR phosphorylation at 2 and $5 \mu \mathrm{M}$. We used $2 \mu \mathrm{M}$ for the remainder of the studies.

Inhibition of PKR reduced NLRP3, caspase 1, and IL$1 \beta$ levels in REC. Once we established the optimal dose of C16, a PKR inhibitor, we investigated whether PKR inhibition regulated the NLRP3 inflammasome pathway in
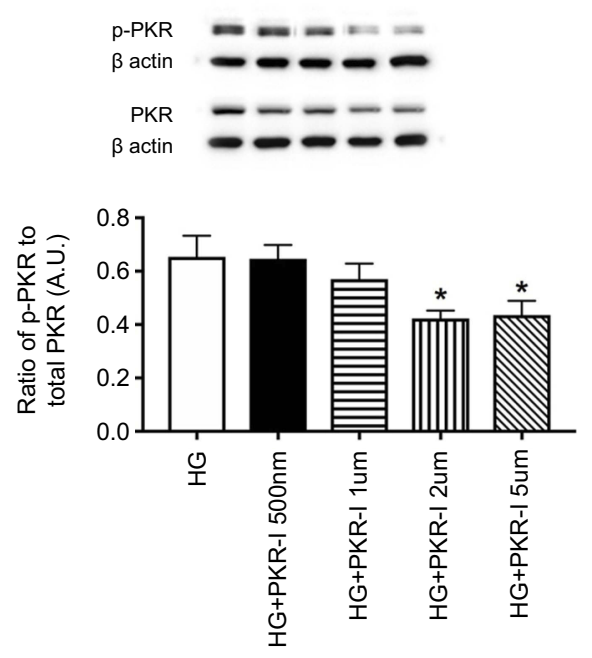

Figure I Dose-response curve for Cl6, a PKR inhibitor (PKR-I). Data is the ratio of phosphorylated PKR to total PKR in retinal endothelial cells (REC) grown in high glucose (HG) or high glucose and $\mathrm{Cl} 6(\mathrm{PKR}-\mathrm{I})$ at $500 \mathrm{nM}, \mathrm{I} \mu \mathrm{M}, 2 \mu \mathrm{M}$, and $5 \mathrm{uM}$. $* P<0.05$ vs $H G$. Data are mean \pm SEM. $N=4$ at each time point.
REC. Figure 2 demonstrates that PKR inhibition significantly reduced PKR (Figure 2A), NLRP3 (Figure 2B), cleaved caspase 1 (Figure 2C) IL-1 $\beta$ Western blot (Figure 2D), and IL-1 $\beta$ ELISA (Figure 2E) in REC grown in high glucose. No changes were noted in cells grown in normal glucose.

Epac1 reduced phosphorylation of PKR both in vivo and in vitro. We have previously reported that Epac1 reduced NLRP3 signaling. ${ }^{11}$ To expand those findings, we used endothelial cell-specific Epac1 knockout mice, as well as REC treated with an Epac1 agonist to determine if Epac1 regulates PKR. Figure 3A shows that Epac1 CreLox mice have significantly increased levels of PKR phosphorylation compared to their floxed littermates. Figure 3B shows similar findings of decreased PKR phosphorylation in REC grown in high glucose that received an Epac1 agonist. Figure $3 \mathrm{C}$ is a control for the mice to demonstrate reduced Epac1 levels in whole retinal lysates from the Epac1 CreLox mice.

Epac1 can overcome PKR to reduce the NLRP3 inflammasome. We next tested whether Epacl could reduce NLRP3 signaling in REC that had been treated with rhPKR. Figure 4A is a time course experiment to show that $48 \mathrm{hrs}$ is effective in increasing PKR phosphorylation in REC grown in high glucose. The images in Figure 4B and $\mathrm{C}$ are controls to demonstrate that the Epac1 agonist (Figure 4B) and rhPKR (Figure 4C) regulated their respective proteins. Figure 4D-F demonstrates that Epac1 reduces NLRP3 (Figure 4D), caspase 1 (Figure 4E), IL-1 $\beta$ Western blot (Figure 4F) levels, and IL-1 $\beta$ ELISA (Figure 4G), while rhPKR increased all 3 proteins. Despite excess PKR, Epac1 continued to decrease NLRP3 signaling when the Epac1 agonist and rhPKR were given at the same time.

\section{Discussion}

An increasing acceptance of the role of inflammation as a causative factor in pre-proliferative diabetic retinopathy has occurred over the past decade. ${ }^{1,3,23}$ More recently, a role for danger associated molecular pattern receptors, specifically HMGB1, has been observed in ocular disease. ${ }^{24,25}$ HMGB1 has been linked to the NLRP3 inflammasome in macrophages. ${ }^{13}$ Since we have also reported that both HMGB1 and NLRP3 are increased in REC, ${ }^{11,20}$ we wanted to investigate mechanisms of activation of NLRP3 in the retina.

While a number of pathways have been reported to activate the NLRP3 inflammasome, we chose to focus this study on PKR. While little has been published on PKR in 
A

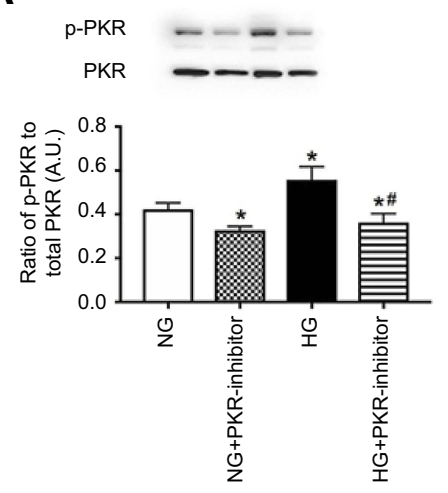

C

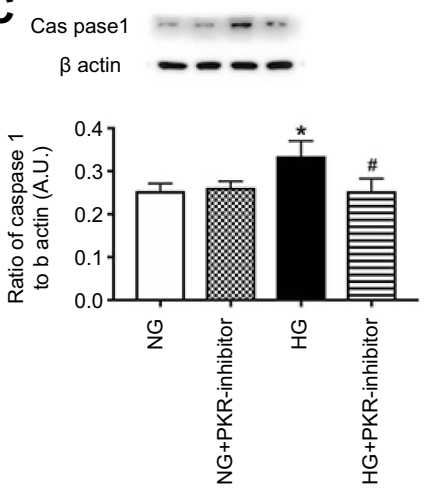

B

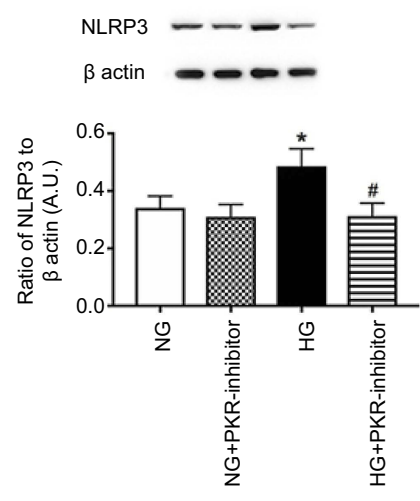

D

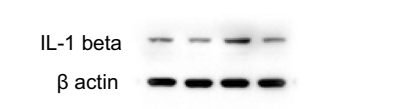

E

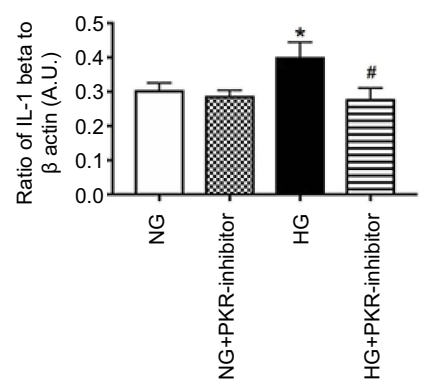

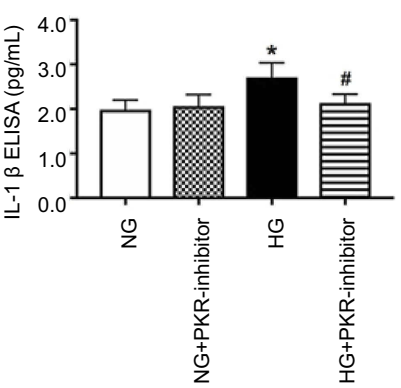

Figure 2 Inhibition of PKR reduces NLRP3 signaling. Retinal endothelial cells (REC) grown in normal glucose (NG) or high glucose (HG) and treated with CI6, a PKR inhibitor (PKR-I). Panel A is a control to show phosphorylated PKR is reduced by the inhibitor. Panel B shows NLRP3 levels, Panel C shows cleaved caspase I (caspasI), Panel $D$ shows IL-I $\beta$ levels by Western blotting, and Panel E shows active IL- I $\beta$ by ELISA in REC. $* P<0.05$ vs $N G$. $\# P<0.05$ vs HG. Data are mean \pm SEM. $N=4$.

the retina or in diabetic retinopathy, a recent review suggested that PKR has a fundamental role in chronic lowgrade inflammation occurring in metabolic disorders, leading to the formation of metainflammation. ${ }^{16}$ Others have reported that inhibition of PKR protects cardiomyocytes against palmitic acid-induced injury ${ }^{26}$ or hydrogen peroxide-induced apoptosis. ${ }^{27}$ Work in osteoblasts suggested that PKR induces expression of NLRP3, leading to periodontal disease. ${ }^{28}$ Work in macrophages is less clear, with studies suggesting that PKR is essential for inducing inducible nitric oxide synthase, but not inflammasomes. ${ }^{29}$ In the eye, one study has shown salvianolic acid A protected RPE cells through inhibition of PKR-NLRP3 signaling. ${ }^{30}$ Thus, literature suggests that in a majority of cells, PKR activates the NLRP3 signaling; however, less is known in diabetic retinopathy.

In these studies, we first tested a PKR inhibitor to investigate whether it could block NLRP3 signaling. C16, a PKR inhibitor, has been reported to prevent inflammation in Alzheimer's disease ${ }^{22,31}$ as well as in an acute excitotoxic rat model. ${ }^{32}$ Our data suggest that $\mathrm{C} 16$ is highly effective in reducing NLRP3 signaling in REC. While C16 was effective, we had previously reported that Epac1 inhibited the NLRP3 inflammasome. ${ }^{11}$ In that study, we focused on HMGB1 and toll-like receptor 4. Literature has linked HMGB1 and PKR with studies showing the reduced PKR levels blocked HMGB1 release. ${ }^{13,19}$ We show that endothelial cell Epac1 knockout mice have increased PKR levels, suggesting that Epac1 can regulate PKR. Further, data in REC grown in high glucose show that an Epac1 agonist can significantly reduce PKR levels. When the Epac1 agonist and recombinant human $\mathrm{PKR}$ are added to REC, data demonstrate that Epac1 overcame excessive PKR levels to reduce activation of the NLRP3 inflammasome. Why recombinant human PKR increased PKR phosphorylation in the REC grown in high glucose is beyond the scope of this study, but can be addressed in the future.

Taken together, our data demonstrate that PKR is involved in NLRP3 activation in REC grown in high glucose. Inhibition of PKR by $\mathrm{C} 16$ or Epac1 was effective in reducing NLRP3 signaling. Data suggest that increased Epac1 signaling in the diabetic retina may reduce activation of the NLRP3 inflammasome via inhibition of PKR. 
A

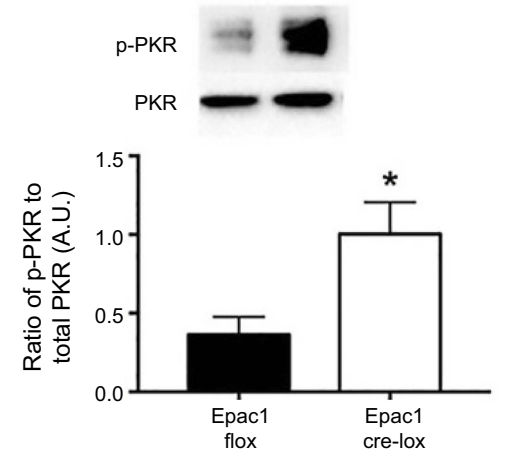

C
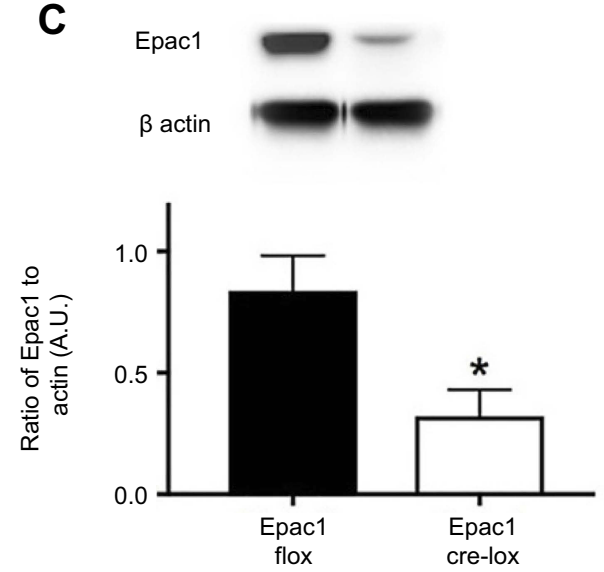

B
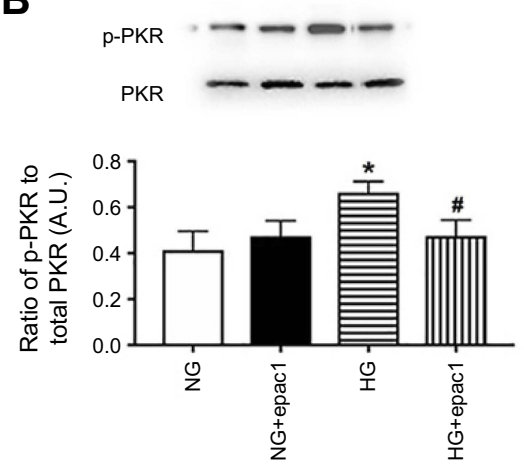

Figure 3 Epacl regulates PKR in retina and in retinal endothelial cells (REC). Panel A shows that PKR phosphorylation is increased in whole retinal lysates from mice where Epacl has been eliminated in endothelial cells. Panel $C$ is a control to show that Epacl is reduced in whole retinal lysates from the retina. Panel $B$ shows that an Epacl agonist can significantly decrease PKR phosphorylation in REC grown in normal glucose (NG) or high glucose (HG). $* P<0.05$ vs $N G$. $\# P<0.05$ vs $H G$. Data are mean $\pm S E M$. N=5 for retina and $\mathrm{N}=4$ for $\mathrm{REC}$.

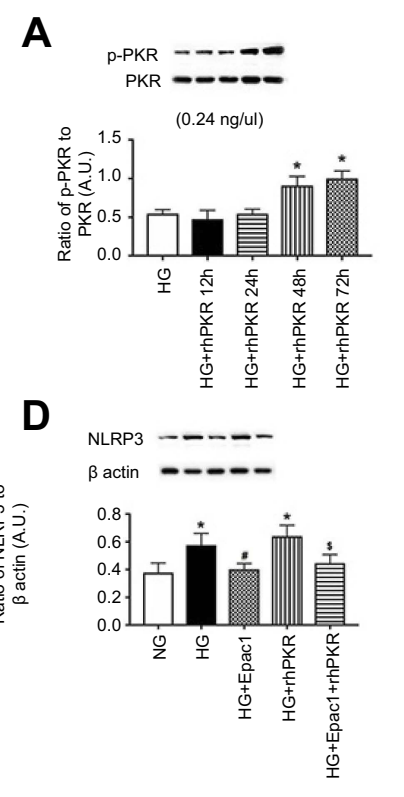

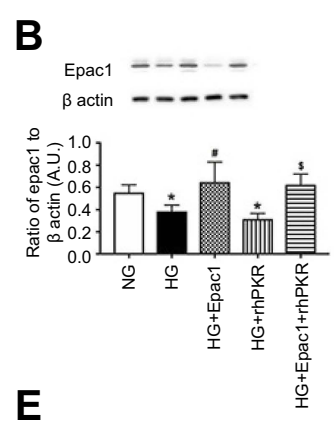

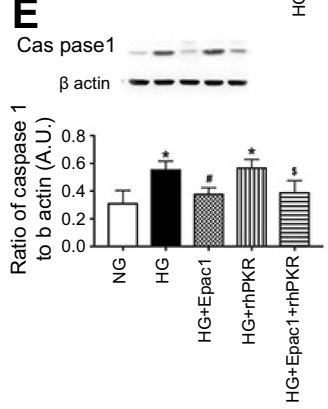

C

Phospho PKR - - -

PKR - - - -

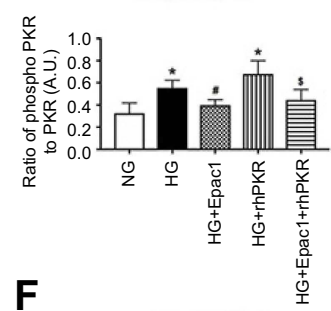

F

IL-1 b - - - -

$\beta$ actin - - -

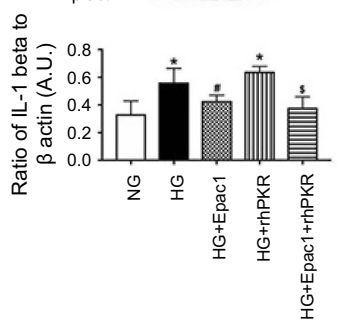

G

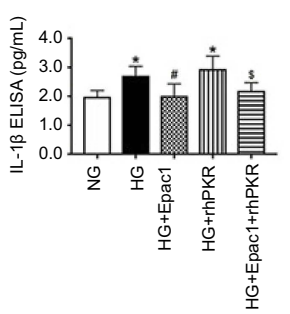

Figure 4 Epacl can overcome recombinant human PKR (rhPKR) to reduce NLRP3 signaling. REC were grown in normal glucose (NG), high glucose (HG) or high glucose treated with the Epacl agonist, rhPKR, and the Epacl agonist+rhPKR. Panel A shows the time course for phosphorylation of PKR with the rhPKR. Panel B shows that the Epacl increased Epacl levels, while Panel $C$ shows that the rhPKR increase PKR levels. Panel D-G shows NLRP3, caspase I and active IL-I $\beta$ levels in the cells with the various treatments. ${ }^{*} P<0.05$ vs $N G$. $\# P<0.05$ vs $H G$. $\$ P<0.05$ vs $H G+$ rhPKR. Data are mean $\pm S E M$. $N=4$. 


\section{Data Availability}

All data is contained in the manuscript.

\section{Acknowledgments}

This study is supported by grants from R01EY028442 (JJS), P30EY04068 (Principal investigator for the core grant: Linda Hazlett), and an unrestricted grant to the Department of Ophthalmology from Research to Prevent Blindness (Kresge Eye Institute). The funders did not influence these designs or execution of these studies.

\section{Author Contributions}

YJ completed the studies. JJS designed the studies, and wrote the draft. Both authors contributed to data analysis, drafting or revising the article, gave final approval of the version to be published, and agree to be accountable for all aspects of the work.

\section{Disclosure}

The authors report no conflicts of interest in this work.

\section{References}

1. Tang J, Kern TS. Inflammation in diabetic retinopathy. Prog Retin Eye Res. 2011;30(5):343-358.

2. Joussen AM, Poulaki V, Le ML, et al. A central role for inflammation in the pathogenesis of diabetic retinopathy. Faseb J. 2004;18 (12):1450-1452. doi:10.1096/fj.03-1476fje

3. Abcouwer SF, Lin CM, Shanmugam S, Muthusamy A, Barber AJ, Antonetti DA. Minocycline prevents retinal inflammation and vascular permeability following ischemia-reperfusion injury. J Neuroinflammation. 2013;10:149. doi:10.1186/1742-2094-10-151

4. Aveleira CA, Lin CM, Abcouwer SF, Ambrosio AF, Antonetti DA. TNF-alpha signals through PKCzeta/NF-kappaB to alter the tight junction complex and increase retinal endothelial cell permeability. Diabetes. 2010;59(11):2872-2882. doi:10.2337/db09-1606

5. Hu P, Thinschmidt JS, Caballero S, et al. Loss of survival factors and activation of inflammatory cascades in brain sympathetic centers in type 1 diabetic mice. Am J Physiol Endocrinol Metab. 2015;308(8): E688-E698. doi:10.1152/ajpendo.00504.2014

6. Grant RW, Dixit VD. Mechanisms of disease: inflammasome activation and the development of type 2 diabetes. Front Immunol. 2013;4:50. doi:10.3389/fimmu.2013.00050

7. Li Y, Liu C, Wan XS, Li SW. NLRP1 deficiency attenuates diabetic retinopathy (DR) in mice through suppressing inflammation response. Biochem Biophys Res Commun. 2018;501(2):351-357. doi:10.1016/j. bbrc.2018.03.148

8. Chen W, Zhao M, Zhao S, et al. Activation of the TXNIP/NLRP3 inflammasome pathway contributes to inflammation in diabetic retinopathy: a novel inhibitory effect of minocycline. Inflamm Res. 2017;66(2):157-166. doi:10.1007/s00011-016-1002-6

9. Chen H, Zhang X, Liao N, et al. Enhanced expression of NLRP3 inflammasome-related inflammation in diabetic retinopathy. Invest Ophthalmol Vis Sci. 2018;59(2):978-985. doi:10.1167/iovs.17-22816

10. Liu L, Jiang Y, Chahine A, Curtiss E, Steinle JJ. Epac1 agonist decreased inflammatory proteins in retinal endothelial cells, and loss of Epac1 increased inflammatory proteins in the retinal vasculature of mice. Mol Vis. 2017;23:1-7.
11. Jiang Y, Liu L, Curtiss E, Steinle JJ. Epac1 blocks NLRP3 inflammasome to reduce IL-1beta in retinal endothelial cells and mouse retinal vasculature. Mediators Inflamm. 2017;2017:2860956. doi:10.1155/ 2017/2860956

12. Wang Y, Hanus JW, Abu-Asab MS, et al. NLRP3 upregulation in retinal pigment epithelium in age-related macular degeneration. Int J Mol Sci. 2016;17:1. doi:10.3390/ijms18010001

13. Lu B, Nakamura T, Inouye K, et al. Novel role of PKR in inflammasome activation and HMGB1 release. Nature. 2012;488 (7413):670-674. doi:10.1038/nature 11290

14. Williams BR. PKR; a sentinel kinase for cellular stress. Oncogene. 1999;18(45):6112-6120. doi:10.1038/sj.onc. 1203127

15. Peters GA, Hartmann R, Qin J, Sen GC. Modular structure of PACT: distinct domains for binding and activating PKR. Mol Cell Biol. 2001;21(6):1908-1920. doi:10.1128/MCB.21.6.1908-1920.2001

16. Sud N, Rutledge AC, Pan K, Su Q. Activation of the dsRNA-activated protein kinase PKR in mitochondrial dysfunction and inflammatory stress in metabolic syndrome. Curr Pharm Des. 2016;22(18):2697-2703.

17. Lourenco MV, Clarke JR, Frozza RL, et al. TNF-alpha mediates PKR-dependent memory impairment and brain IRS-1 inhibition induced by Alzheimer's beta-amyloid oligomers in mice and monkeys. Cell Metab. 2013;18(6):831-843. doi:10.1016/j. cmet.2013.11.002

18. Kang R, Tang D. PKR-dependent inflammatory signals. Sci Signal. 2012;5(247):pe47. doi:10.1126/scisignal.2003289

19. Lu B, Wang H, Andersson U, Tracey KJ. Regulation of HMGB1 release by inflammasomes. Protein Cell. 2013;4(3):163-167. doi:10.1007/s13238-012-2118-2

20. Liu L, Jiang Y, Steinle JJ, Ramchandran R. Epac1 protects the retina against ischemia/reperfusion-induced neuronal and vascular damage. PLoS One. 2018;13(9):e0204346. doi:10.1371/journal.pone.0204346

21. Curtiss E, Jiang Y, Liu L, Hawthorne C, Zhang J, Steinle JJ. Epac1 restores normal insulin signaling through a reduction in inflammatory cytokines. Mediators Inflamm. 2018;2018:3809092. doi:10.1155/ 2018/3809092

22. Couturier J, Morel M, Pontcharraud R, et al. Interaction of double-stranded RNA-dependent protein kinase (PKR) with the death receptor signaling pathway in amyloid beta (Abeta)-treated cells and in APPSLPS1 knock-in mice. J Biol Chem. 2010;285 (2):1272-1282. doi:10.1074/jbc.M109.041954

23. Koizumi K, Poulaki V, Doehmen S, et al. Contribution of TNF-alpha to leukocyte adhesion, vascular leakage, and apoptotic cell death in endotoxin-induced uveitis in vivo. Invest Ophthalmol Vis Sci. 2003;44(5):2184-2191. doi:10.1167/iovs.02-0589

24. Ekanayaka SA, McClellan SA, Barrett RP, Kharotia S, Hazlett LD. Glycyrrhizin reduces HMGB1 and bacterial load in pseudomonas aeruginosa keratitis. Invest Ophthalmol Vis Sci. 2016;57 (13):5799-5809. doi:10.1167/iovs.16-20103

25. Zhang J, Wu Y, Weng Z, Zhou T, Feng T, Lin Y. Glycyrrhizin protects brain against ischemia-reperfusion injury in mice through HMGB1-TLR4-IL-17A signaling pathway. Brain Res. 2014;1582:176-186. doi:10.1016/j.brainres.2014.07.002

26. Mangali S, Bhat A, Udumula MP, Dhar I, Sriram D, Dhar A. Inhibition of protein kinase $\mathrm{R}$ protects against palmitic acid-induced inflammation, oxidative stress, and apoptosis through the JNK/ NF-kB/NLRP3 pathway in cultured H9C2 cardiomyocytes. $J$ Cell Biochem. 2019;120(3):3651-3663. doi:10.1002/jcb.27643

27. Wang Y, Men M, Xie B, et al. Inhibition of PKR protects against $\mathrm{H} 2 \mathrm{O} 2$-induced injury on neonatal cardiac myocytes by attenuating apoptosis and inflammation. Sci Rep. 2016;6:38753. doi:10.1038/ srep38753

28. Yoshida K, Okamura H, Hiroshima Y, et al. PKR induces the expression of NLRP3 by regulating the NF-kappaB pathway in porphyromonas gingivalis-infected osteoblasts. Exp Cell Res. 2017;354 (1):57-64. doi:10.1016/j.yexcr.2017.03.028 
29. He Y, Franchi L, Nunez G. The protein kinase PKR is critical for LPS-induced iNOS production but dispensable for inflammasome activation in macrophages. Eur J Immunol. 2013;43(5):1147-1152. doi:10.1002/eji.201243187

30. Mao K, Shu W, Qiu Q, Gu Q, Wu X. Salvianolic acid A protects retinal pigment epithelium from OX-LDL-induced inflammation in an age-related macular degeneration model. Discov Med. 2017;23 (125):129-147.
31. Couturier J, Paccalin M, Lafay-Chebassier C, et al. Pharmacological inhibition of PKR in APPswePS1dE9 mice transiently prevents inflammation at 12 months of age but increases Abeta42 levels in the late stages of the Alzheimer's disease. Curr Alzheimer Res. 2012;9(3):344-360.

32. Tronel C, Page G, Bodard S, Chalon S, Antier D. The specific PKR inhibitor $\mathrm{C} 16$ prevents apoptosis and IL-1beta production in an acute excitotoxic rat model with a neuroinflammatory component. Neurochem Int. 2014;64:73-83. doi:10.1016/j.neuint.2013.10.012

\section{Publish your work in this journal}

The Journal of Inflammation Research is an international, peerreviewed open-access journal that welcomes laboratory and clinical findings on the molecular basis, cell biology and pharmacology of inflammation including original research, reviews, symposium reports, hypothesis formation and commentaries on: acute/chronic inflammation; mediators of inflammation; cellular processes; molecular mechanisms; pharmacology and novel anti-inflammatory drugs; clinical conditions involving inflammation. The manuscript management system is completely online and includes a very quick and fair peerreview system. Visit http://www.dovepress.com/testimonials.php to read real quotes from published authors. 